\title{
Three-body force from kinematical effects in the three-nucleon system
}

\author{
Ch. Hajduk and P. U. Sauer \\ Theoretical Physics, Universität Hannover, 3000 Hannover, Germany
}

(Received 29 January 1980)

\begin{abstract}
A Galilean-noninvariant correction to ordinary one-boson-exchange potentials is derived. It gives rise to a threebody force in the three-nucleon system. Its importance for the properties of the three-nucleon bound state is tested and found to be small. This result justifies the common practice which neglects the dependence of the one-bosonexchange potentials on the total momentum of the interacting pair in many-nucleon systems.
\end{abstract}

[NUCLEAR STRUCTURE Three-body gound state dependence of nucleon-nucle-
on potential on total pair momentum.

A two-body potential arising from particle exchange has a part which depends on the total momentum of the interacting pair $\overrightarrow{\mathrm{P}}_{\mathrm{c} . \mathrm{m} .}$. This Galilean-noninvariant piece of the potential is known for the one-photon exchange and is usually taken into account when the Breit interaction ${ }^{1}$ is applied. The corresponding piece of the two-nucleon oneboson-exchange potential has never been employed yet. It is unnecessary when the potential $v\left(\overrightarrow{\mathrm{P}}_{\mathrm{c} . \mathrm{m} .}\right)$ is fitted to the two-nucleon data, since in this case a coordinate system can always be chosen in which the total pair momentum is zero. The Galileannoninvariant part of the one-boson-exchange potential, i.e.,

$$
\delta v\left(\overrightarrow{\mathrm{P}}_{\mathrm{c} . \mathrm{m} .}\right)=v\left(\overrightarrow{\mathrm{P}}_{\mathrm{c} . \mathrm{m} .}\right)-v(0),
$$

will only show up in the description of a many-nucleon system. In this paper we explore its importance and choose as an example its effects on the three-nucleon bound state. What is the interest in this particular case?

The three-nucleon bound state is considered for vanishing overall c.m. momentum. In this case the total momentum of the interacting pair $\overrightarrow{\mathrm{P}}_{\mathrm{c} . \mathrm{m} \text {. }}$ equals $-\vec{q}$, the negative momentum of the spectator nucleon. The Galilean-noninvariant part of the oneboson-exchange potential therefore depends on the relative momentum $\vec{p}$ of the interacting nucleon pair and on the spectator momentum $\vec{q}$. Therefore, it is a qenuine three-body force $\left\langle\overrightarrow{\mathrm{p}}^{\prime} \overrightarrow{\mathrm{q}}^{\prime}|V| \overrightarrow{\mathrm{pq}}\right\rangle$, i.e.,

$$
\left\langle\overrightarrow{\mathrm{p}}^{\prime} \overrightarrow{\mathrm{q}}^{\prime}|V| \overrightarrow{\mathrm{p}} \overrightarrow{\mathrm{q}}\right\rangle=\delta\left(\overrightarrow{\mathrm{q}}^{\prime}-\overrightarrow{\mathrm{q}}\right)\left\langle\overrightarrow{\mathrm{p}}^{\prime}|v(-\overrightarrow{\mathrm{q}}\rangle| \overrightarrow{\mathrm{p}}\right\rangle \text {. }
$$

As will become evident from its explicit form, it is repulsive when the spectator nucleon approaches the three-body c.m., even for fairly large spatial separations of the interacting pair. This spatial distribution of repulsive potential energy is quite different from the way the repulsive cores of the two-nucleon potentials act, and therefore deserves investigation. Next, the explicit form of the threebody force is given, then its effect on the ${ }^{3} \mathrm{H}$ bind- ing energy and the ${ }^{3} \mathrm{He}$ charge form factor is presented, and finally the physical implications of the results are discussed.

The ordinary derivation ${ }^{2}$ of the one-boson-exchange potentials is redone assuming nonvanishing pair momentum $\overrightarrow{\mathrm{P}}_{\mathrm{c} . \mathrm{m} \text {. }}$ as in Fig. 1. The derivation is not carried out in helicity representation; the spin dependence of the coupling vertices is kept in operator form. The derivation is sketched in the Appendix. Scalar and vector coupling yield the following contributions to $\delta v\left(\overrightarrow{\mathrm{P}}_{\mathrm{c} . \mathrm{m} .}\right)$ up to the order $\left[\overrightarrow{\mathrm{P}}_{\text {c. m. }} /(M c)\right]^{2}$ :

$$
\begin{aligned}
\left\langle\overrightarrow{\mathrm{p}}^{\prime}\left|\delta v\left(\overrightarrow{\mathrm{P}}_{\text {c. m. }}\right)\right| \overrightarrow{\mathrm{p}}\right\rangle= & ( \pm) \frac{g^{2}}{4 \pi} \frac{1}{\left(\overrightarrow{\mathrm{p}}^{\prime}-\overrightarrow{\mathrm{p}}\right)^{2} c^{2}+m^{2} c^{4}} \\
& \times \frac{1}{8}\left(\frac{\overrightarrow{\mathrm{P}}_{\text {c.me }}}{M c}\right)^{2} .
\end{aligned}
$$

The plus (minus) sign holds for scalar (vector) coupling, $g^{2}$ is the coupling constant, $m$ the mass of the exchanged meson, and $M$ the nucleon mass. The meson parameters used are listed in Table I. There is no contribution from pseudoscalar coupling. The contributions of Eq. (3) arise from the coupling vertices, not from the meson propagator.

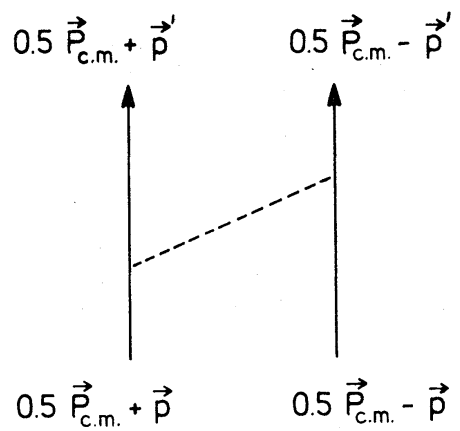

FIG. 1. Notation of the momenta used for the description of the one-boson-exchange potential. 
TABLE I. Meson parameters used for the Galilean noninvariant contribution in the one-boson-exchange potential. The parameters are taken from Ref. 3. $J^{P}$ and $T$ denote the spin, parity, and isospin of the meson.

\begin{tabular}{cccrrrl}
\hline \hline Meson & $J^{P}$ & $T$ & $m(\mathrm{MeV})$ & $g^{2} / 4 \pi$ & {$\left[(\mathrm{MeV} \mathrm{fm})^{3}\right]$} & $f / g$ \\
\hline$\sigma$ & $0^{+}$ & 0 & 520 & 5.66 & 0 \\
$\delta$ & $0^{+}$ & 1 & 960 & 0.82 & 0 \\
$\rho$ & $1^{-}$ & 1 & 711 & 0.50 & 6.2 \\
$\omega$ & $1^{-}$ & 0 & 783 & 10.00 & 0 \\
$\phi$ & $1^{-}$ & 0 & 1020 & 0.00 & 0 \\
\hline \hline
\end{tabular}

In the same order $(M c)^{-2}$ there are also (partly spin dependent) cross terms of the form $\overrightarrow{\mathrm{p}} \cdot \overrightarrow{\mathrm{P}}_{\mathrm{c} . \mathrm{m}}$

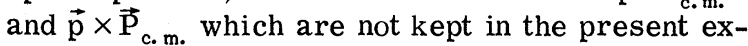
ploration. Their effect is hoped to be suppressed due to an effective averaging over the angle between $\vec{p}$ and $\vec{P}_{\text {c.m. }}$. in the nuclear many-body system. The correction potentials $\delta v\left(\overrightarrow{\mathbf{P}}_{\text {c.m. }}\right)$ are displayed in Figs. 2 and 3 in a mixed representation. As compared to the ordinary two-body potentials there is a reversed spatial distribution of repulsion and attraction. The potentials $\delta v\left(\overrightarrow{\mathrm{P}}_{\mathrm{c} . \mathrm{m} .}\right)$ are relativistic corrections. We are well aware that

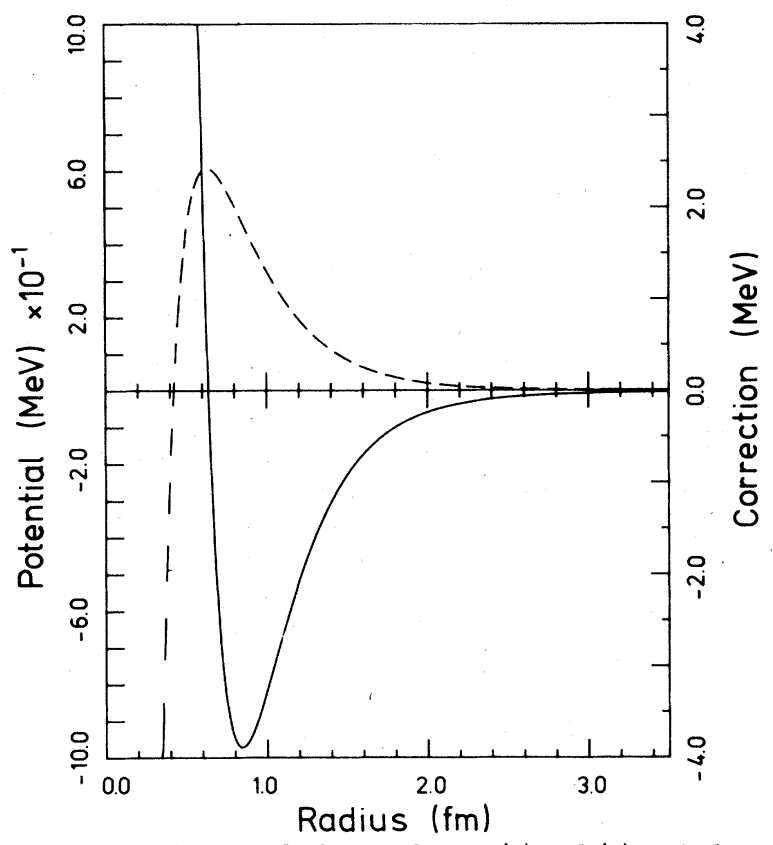

FIG. 2. Three-body force of Eqs. (2) and (3)'with the parameters of Table $I$ in a mixed representation, i.e., $\left.\left.\left\langle\overrightarrow{\mathrm{r}}^{\prime}\right| \delta v(-\overrightarrow{\mathrm{q}})\right] \overrightarrow{\mathrm{r}}\right\rangle=\delta\left(\overrightarrow{\mathrm{r}}^{\prime}-\overrightarrow{\mathrm{r}}\right) v(r, q)$. The nucleon pair interacts in ${ }^{1} S_{0}$, the corresponding relative distance is $\overrightarrow{\mathrm{r}}$. The potential is shown as dotted curve for $q=\frac{1}{2} M c$, a value close to the cutoff $q_{\max }$ in the solution of the Faddeev equations. In coordinate space the potential is nonlocal with respect to the distance of the spectator from the c.m. of the pair. The right scale refers to $v(r, q)$. The ${ }^{1} S_{0}$ Reid soft-core potential is shown for comparison as solid curve, the left scale refers to it.

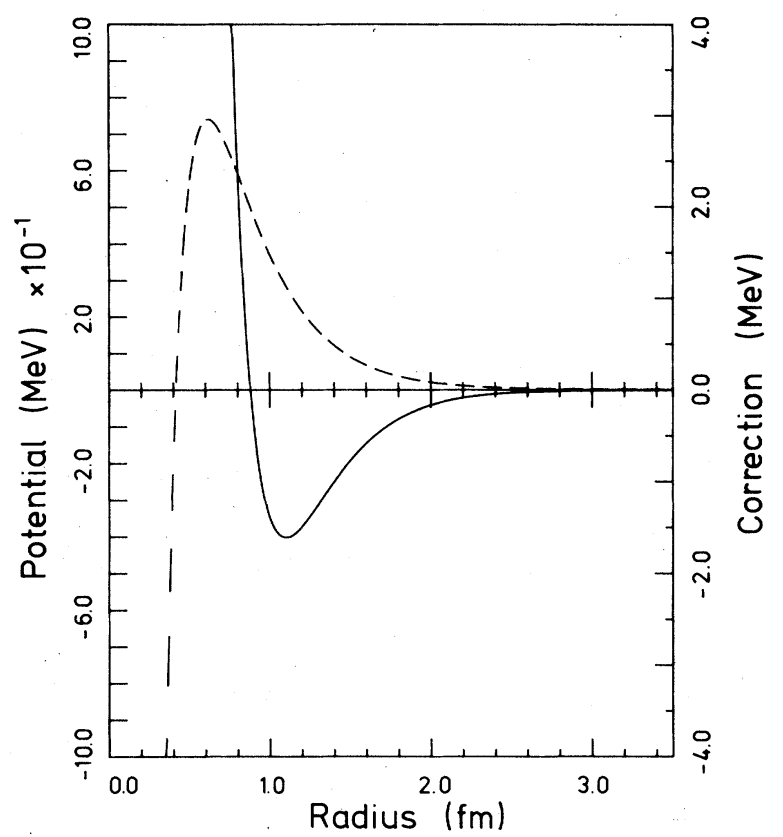

FIG. 3. Three-body force of Eqs. (2) and (3) and of Table I in configuration space. The nucleon pair interacts in ${ }^{3} S_{1}$. The ${ }^{3} S_{1}$ Reid soft-core potential is shown for comparison as solid curve. For details see Fig. 2 caption.

the naive derivation of $\delta v\left(\overrightarrow{\mathrm{P}}_{\mathrm{c} . \mathrm{m} .}\right)$ does not yield the relativistic corrections in a systematic way. However, it clearly yields an order of magnitude estimate sufficient for the present investigation. If need arises for an improved derivation, it should follow the ideas of Ref. 4.

The three-body force of Eqs. (2) and (3) is used in a calculation of the three-nucleon bound state. The Reid soft-core potential ${ }^{5}$ in the partial waves ${ }^{1} S_{0}$ and ${ }^{3} S_{1}-{ }^{3} D_{1}$ is taken as the standard two-nucleon interaction $v\left(\overrightarrow{\mathbf{P}}_{\text {c.m. }}=0\right)$. An ordinary threebody potential $\left\langle\overrightarrow{\mathrm{p}}^{\prime} \overrightarrow{\mathrm{q}}^{\prime}|V| \overrightarrow{\mathrm{pq}}\right\rangle$ is regular in three-body space and therefore must be treated differently from two-body potentials in the Faddeev equations for the three-nucleon bound state. The special three-body force of Eqs. (2) and (3) considered here has, however, with respect to the spectator momentum $\vec{q}$, the same singular behavior as a two-body potential. It can therefore be treated on the same level as the two-body potential. It is summed together with the two-body potential into a two-body transition matrix, whose parametrical dependence on the spectator momentum now arises from the potential as well as from the three-body Green's function.

Thus, the three-body for ce of Eqs. (2) and (3) is included in a calculation of the three-nucleon bound state without any technical complication as it stands, taking into account all three possible combinations of interacting pairs and spectating nucleons. The momentum cutoff for the spectator 
momentum $\overrightarrow{\mathrm{q}}$, used when solving the Faddeev equations, ${ }^{6}$ i.e., $q_{\max }=3.6 \mathrm{fm}^{-1}$ for the Jacobi coordinate $q$, prevents the three-body force from being used for large $q$ when its behavior becomes irregular. Consistent with the spatial shape of the threebody potential, shown in Figs. 2 and 3, its effect on the binding energy is repulsive, but the repulsive effect is very small. The inclusion of the three-body force decreases the binding energy by $0.05 \mathrm{MeV}$ compared to a calculation based on the Reid soft-core potential without the three-body force. The relative changes in the ${ }^{3} \mathrm{He}$ charge form factor are up to momentum transfers $Q^{2}$ $=100 \mathrm{fm}^{-2}$, outside the diffraction minima, always smaller than $10^{-2}$, usually of the order of $10^{-3}$. The diffraction minima shift by less than $0.1 \mathrm{fm}^{-2}$. We also note that the used meson parameters of Table I refer to a one-boson-exchange potential with eikonal form factors. In contrast, the meson parameters of Ref. 7 decrease the overall size of the three-body force $\delta v$ in the spatial region of importance. In the latter parameter set the coup- ling constants of the vector mesons are comparatively large. The attraction in $\delta v$ at small relative distances is therefore increased as compared to Figs. 2 and 3 , pushing the zero of $\delta v$ out to $1 \mathrm{fm}$ and simultaneously reducing the maximum repulsion by a factor of 10 . Thus, our calculation can even be interpreted to yield an upper bound to the effect of the studied three-body force $\delta v$.

We conclude that the studied three-body force affects the properties of the three-nucleon bound state in an entirely insignificant way. We do not take this as a negative result, but rather as a gratifying illustrative proof, that the standard procedure, which uses the one-boson-exchange potentails with $\overrightarrow{\mathrm{P}}_{\text {c. } \mathrm{m} .}=0$ also in a many-nucleon system, is quite well founded.

The authors are grateful to $\mathrm{K}$. Holinde for useful discussions on this problem and for his help in deriving the results of Eq. (3). The calculations were performed at Regionales Rechenzentrum für Niedersachsen. The work was supported by funds of the Deutsche Forschungsgemeinschaft (DFG).

\section{APPENDIX}

The potential corresponding to the one-boson-exchange process of Fig. 1 is given by the following matrix element

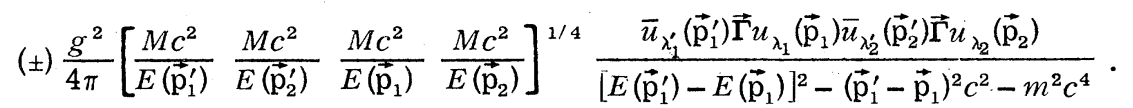

In Eq. (A1) $u_{\lambda_{i}}\left(\overrightarrow{\mathrm{p}}_{i}\right)$ denotes the positive-energy Dirac spinor with the normalization $\bar{u}_{\lambda_{i}}(\overrightarrow{\mathrm{p}}) \cdot u_{\lambda_{j}}(\overrightarrow{\mathrm{p}})=\delta_{\lambda_{i} \lambda_{j}}, \vec{\Gamma}$ the meson-nucleon vertex, $\overrightarrow{\mathrm{p}}_{1}=\frac{1}{2} \overrightarrow{\mathrm{P}}_{\mathrm{c} . \mathrm{m} .}+\overrightarrow{\mathrm{p}}, \overrightarrow{\mathrm{p}}_{2}=\frac{1}{2} \overrightarrow{\mathrm{P}}_{\mathrm{c} . \mathrm{m} .}-\overrightarrow{\mathrm{p}}$, and $E\left(\overrightarrow{\mathrm{p}}_{i}\right)=\left(M c^{2}+p_{i}{ }^{2} c^{2}\right)^{1 / 2}$. The plus (minus) sign holds for scalar and pseudoscalar (vector) couplings. The spinor normalization factors, i.e., the power $\frac{1}{4}$ of the square bracket, appears strangely chosen. With this choice, however, the matrix element (A1) reduces in the two-body c.m. system to that form of the potential, which makes ${ }^{2}$ the Blankenbecler-Sugar approximation to the Bethe-Salpeter equation formally identical with the Lippmann-Schwinger equation. Thus, the potential (A1) is to be employed together with the nonrelativistic kinetic-energy operator. In this way it is used in the calculation of this paper.

In contrast to (A1), the ordinary derivation ${ }^{8}$ of the Breit interaction-the original motivation for the present work-interprets the Born term in the perturbation expansion for the $S$ matrix as potential. This Born term differs from (A1) by spinor normalization factors, i.e., it contains the power $\frac{1}{2}$ for the square bracket, and it should be used ${ }^{8}$ together with a relativistically corrected kinetic-energy operator. The resulting potential would be larger by a factor of 2 than that of Eq. (3) without changing the conclusion of this paper.

For the explicit evaluation of the potential (A1) the spinors are needed in the combination $u_{\lambda_{i}}\left(\overrightarrow{\mathrm{p}}_{i}\right)\left[M c^{2} / E\left(\overrightarrow{\mathrm{p}}_{i}\right)\right]^{1 / 4}$. They are expanded up to the order $(M c)^{-2}$, i.e.,

$$
u_{\lambda_{i}}\left(\overrightarrow{\mathrm{p}}_{i}\right)\left[\frac{M c^{2}}{E\left(\overrightarrow{\mathrm{p}}_{i}\right)}\right]^{1 / 4}=\left(\frac{\vec{\sigma}_{i} \cdot \overrightarrow{\mathrm{p}}_{i}}{2 M c}\right) \chi_{\lambda_{i}}
$$

$\chi_{\lambda_{i}}$ being two-component Pauli spinors. The vertices

$$
\bar{u}_{\lambda_{i}}\left(\overrightarrow{\mathrm{p}}_{i}^{\prime}\right) \vec{\Gamma} u_{\lambda_{i}}\left(\overrightarrow{\mathrm{p}}_{i}\right)\left[\frac{M c^{2}}{E\left(\overrightarrow{\mathrm{p}}_{i}^{\prime}\right)} \frac{M c^{2}}{E\left(\overrightarrow{\mathrm{p}}_{i}\right)}\right]^{1 / 4}
$$

in Eq. (A1) are calculated up to the same order $(M c)^{-2}$ and their dependence on the c.m. momentum $\overrightarrow{\mathrm{P}}_{c . m}$. is made explicit: 


$$
\begin{aligned}
& \bar{u}_{\lambda_{i}^{\prime}}\left(\overrightarrow{\mathrm{p}}_{i}^{\prime}\right) u_{\lambda_{i}}\left(\overrightarrow{\mathrm{p}}_{i}\right)\left[\frac{M c^{2}}{E\left(\overrightarrow{\mathrm{p}}_{i}^{\prime}\right)} \frac{M c^{2}}{E\left(\overrightarrow{\mathrm{p}}_{i}\right)}\right]^{1 / 4}=\chi_{\lambda_{i}}^{T}\left\{1-\left(\frac{1}{2 M c}\right)^{2}\left[\overrightarrow{\mathrm{p}}^{\prime} \cdot \overrightarrow{\mathrm{p}}+i \vec{\sigma}_{i}\left(\overrightarrow{\mathrm{p}}^{\prime} \times \overrightarrow{\mathrm{p}}\right)_{ \pm} \frac{1}{2}\left(\overrightarrow{\mathrm{p}}^{\prime}+\overrightarrow{\mathrm{p}}\right) \cdot \overrightarrow{\mathrm{p}}_{\mathrm{c}, \mathrm{m} .}\right.\right.
\end{aligned}
$$

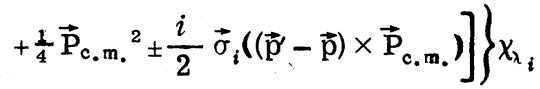

$$
\begin{aligned}
& \bar{u}_{\lambda_{i}^{\prime}}\left(\overrightarrow{\mathrm{p}}_{i}^{\prime}\right) \gamma_{5} u_{\lambda_{i}}\left(\overrightarrow{\mathrm{p}}_{i}\right)\left[\frac{M c^{2}}{E\left(\overrightarrow{\mathrm{p}}_{i}^{\prime}\right)} \frac{M c^{2}}{E\left(\overrightarrow{\mathrm{p}}_{i}^{\prime}\right)}\right]^{1 / 4}=\chi_{\lambda_{i}}^{T} \frac{( \pm i)}{2 M c} \vec{\sigma}_{i}(\overrightarrow{\mathrm{p}}-\overrightarrow{\mathrm{p}}) \chi_{\lambda_{i}}, \\
& \bar{u}_{\lambda_{i}^{\prime}}\left(\overrightarrow{\mathrm{p}}_{i}^{\prime}\right) \gamma_{0} u_{\lambda_{i}}\left(\overrightarrow{\mathrm{p}}_{i}\right)\left[\frac{M c^{2}}{E\left(\overrightarrow{\mathrm{p}}_{i}^{\prime}\right)} \frac{M c^{2}}{E\left(\overrightarrow{\mathrm{p}}_{i}\right)}\right]^{1 / 4}=\chi_{\lambda_{i}^{\prime}}^{T}\left\{1+\left(\frac{1}{2 M c}\right)^{2}\left[\overrightarrow{\mathrm{p}} \cdot \overrightarrow{\mathrm{p}}+i \vec{\sigma}_{i}\left(\overrightarrow{\mathrm{p}}^{\prime} \times \overrightarrow{\mathrm{p}}\right)_{ \pm} \pm \frac{1}{2}(\overrightarrow{\mathrm{p}}+\overrightarrow{\mathrm{p}}) \cdot \overrightarrow{\mathrm{p}}_{\mathrm{c}, \mathrm{m} .}\right.\right. \\
& \left.\left.+\frac{1}{4} \overrightarrow{\mathrm{P}}_{\mathrm{c} . \mathrm{m} .}{ }^{2} \pm \frac{i}{2} \vec{\sigma}_{i}\left((\overrightarrow{\mathrm{p}}-\overrightarrow{\mathrm{p}}) \times \overrightarrow{\mathrm{P}}_{\mathrm{c} \cdot \mathrm{m} .}\right)\right]\right\} X_{\lambda_{i}}, \\
& u_{\lambda_{i}^{\prime}}\left(\overrightarrow{\mathrm{p}}_{i}^{\prime}\right) \vec{\gamma} u_{\lambda_{i}}\left(\overrightarrow{\mathrm{p}}_{i}\right)\left[\frac{M c^{2}}{E\left(\overrightarrow{\mathrm{p}}_{i}\right)} \frac{M c^{2}}{E\left(\overrightarrow{\mathrm{p}}_{i}\right)}\right]^{1 / 4}=\chi_{\lambda_{i}^{\prime}}^{T} \frac{1}{2 M c}\left[ \pm \overrightarrow{\mathrm{p}}^{\prime} \pm \overrightarrow{\mathrm{p}}_{ \pm i \vec{\sigma}_{i}} \times\left(\overrightarrow{\mathrm{p}}^{\prime}-\overrightarrow{\mathrm{p}}\right)+\overrightarrow{\mathrm{P}}_{\mathrm{c}, \mathrm{m} .}\right] \chi_{\lambda_{i}} \cdot
\end{aligned}
$$

The upper (lower) sign refers to $i=1(2)$. The meson propagator takes the form

$$
\frac{1}{\left[E\left(\overrightarrow{\mathrm{p}}_{1}^{\prime}\right)-E\left(\overrightarrow{\mathrm{p}}_{1}\right)\right]^{2}-\left(\overrightarrow{\mathrm{p}}_{1}^{\prime}-\overrightarrow{\mathrm{p}}_{1}\right)^{2} c^{2}-m^{2} c^{4}}=\frac{(-1)}{\left(\overrightarrow{\mathrm{p}}_{1}^{\prime}-\overrightarrow{\mathrm{p}}_{1}\right)^{2} c^{2}+m^{2} c^{4}}\left\{1-\left(\frac{1}{2 M c}\right)^{2}\left[\frac{\left(\overrightarrow{\mathrm{p}}^{2}-\overrightarrow{\mathrm{p}}^{2}\right)^{2}}{\left(\overrightarrow{\mathrm{p}}^{\prime}-\overrightarrow{\mathrm{p}}^{2}+m^{2} c^{2}\right.}-\frac{\left.\left(\left(\overrightarrow{\mathrm{p}}^{\prime}-\overrightarrow{\mathrm{p}}\right) \cdot \overrightarrow{\mathrm{p}}_{c \cdot m}\right)^{2}\right)}{\left(\overrightarrow{\mathrm{p}}^{\prime}-\overrightarrow{\mathrm{p}}^{2}\right)^{2}+m^{2} c^{2}}\right]\right\} .
$$

The results (A3)-(A7), when inserted into (A1), yield Eq. (3), provided the following approximations are made: (i) Terms of the order $(M c)^{-3}$ and higher are neglected. (ii) It is assumed, though numerically not checked, that the c.m. dependences $\left(\vec{p}^{\prime} \pm \vec{p}\right) \cdot \overrightarrow{\mathrm{p}}_{c, m}$. and $\left(\vec{p}^{\prime}-\vec{p}\right) \times \overrightarrow{\mathrm{P}}_{c, m}$. of order $(M c)^{-2}$ are effectively averaged to zero in the many-nucleon system. (iii) Since a correction in $\overrightarrow{\mathrm{P}}_{\mathrm{c} . \mathrm{m}}$. is calculated, the nonrelativistic form of the propagator is used, i. e., the term $\left[1 /(2 M c)^{2}\right]\left(\vec{p}^{\prime}-\vec{p}^{2}\right)^{2} /\left[(\vec{p}-\vec{p})^{2}+m^{2} c^{2}\right]^{2}$ is also dropped. According to (A4) there is no contribution to $\delta v\left(\overrightarrow{\mathrm{P}}_{\mathrm{c}, \mathrm{m}}\right)$ from pseudoscalar coupling. For the vector coupling case the gradient contribution is included. There is, however, no dependence of $\delta v\left(\overrightarrow{\mathrm{P}}_{\mathrm{c} . \mathrm{m} .}\right)$ on the coupling constant $f$.

${ }^{1}$ F. Close and H. Osborn, Phys. Rev. D 2, 2127 (1970).

${ }^{2}$ K. Erkelenz, Phys. Rep. 13C, $191(197 \overrightarrow{4})$.

${ }^{3} \mathrm{~K}$. Holinde and R. Machleidt, Nucl. Phys. A256, 479 (1976).

${ }^{4} \mathrm{~J}$. L. Friar, in Mesons in Nuclei, edited by M. Rho and D. Wilkinson (North-Holland, Amsterdam, 1979), p. 595.

${ }^{5}$ R. V. Reid, Ann. Phys. (N.Y.) 50, 411 (1968).

${ }^{6}$ R. A. Malfliet and J. A. Tjon, Ann. Phys. (N.Y.) 61 ,
425 (1970); E. P. Harper, Y. E. Kim, and A. Tubis, Phys. Rev. Lett. 28, 1533 (1972); our own slight variation of the computational technique is given in $\mathrm{Ch}$. Hajduk and P. U. Sauer, Nucl. Phys. A322, 329 (1979). ${ }^{7}$ K. Erkelenz, K. Holinde, and R. Machleidt, Phys. Lett. 49B, 209 (1974).

${ }^{8} \mathrm{~L} . \mathrm{D}$. Landau and E. M. Lifschitz, Course of Theoretical Physics (Pergamon, Oxford, 1971), Vol. 4, part 1, p. 280 . 